\title{
Rapid control of ectopic Cushing's syndrome during the COVID-19 pandemic in a patient with chronic hypokalaemia
}

\author{
Ziadoon Faisal1 and Miguel Debono D2 \\ 1Department of General Medicine, Royal Victoria Infirmary, Newcastle Upon Tyne Hospitals NHS Foundation Trust, \\ Newcastle Upon Tyne, UK and 'Department of Diabetes and Endocrinology, Royal Hallamshire Hospital, Sheffield \\ Teaching Hospitals NHS Foundation Trust, Sheffield, UK
}

Correspondence

should be addressed

to M Debono

Email

miguel.debono@nhs.net

\section{Summary}

In this case report, we describe the management of a patient who was admitted with an ectopic ACTH syndrome during the COVID pandemic with new-onset type 2 diabetes, neutrophilia and unexplained hypokalaemia. These three findings when combined should alert physicians to the potential presence of Cushing's syndrome (CS). On admission, a quick diagnosis of CS was made based on clinical and biochemical features and the patient was treated urgently using high dose oral metyrapone thus allowing delays in surgery and rapidly improving the patient's clinical condition. This resulted in the treatment of hyperglycaemia, hypokalaemia and hypertension reducing cardiovascular risk and likely risk for infection. Observing COVID-19 pandemic international guidelines to treat patients with CS has shown to be effective and offers endocrinologists an option to manage these patients adequately in difficult times.

\section{Learning points:}

- This case report highlights the importance of having a low threshold for suspicion and investigation for Cushing's syndrome in a patient with neutrophilia and hypokalaemia, recently diagnosed with type 2 diabetes especially in someone with catabolic features of the disease irrespective of losing weight.

- It also supports the use of alternative methods of approaching the diagnosis and treatment of Cushing's syndrome during a pandemic as indicated by international protocols designed specifically for managing this condition during Covid-19.

\section{Background}

The COVID-19 pandemic has hugely influenced medical practice. To maintain social isolation and lockdown restrictions, authorities have modified standard protocols to reduce COVID-19 spread. A typical complex condition influenced by these changes has been Cushing's syndrome (CS). We present a case of a patient with persisting neutrophilia and hypokalaemia diagnosed with ectopic ACTH syndrome from small cell lung cancer with sacral metastases. The patient had recently been diagnosed with type 2 diabetes; irrespective of clinical and biochemical features of CS, the diagnosis was delayed.

Supported by COVID-19 international protocol modifications, routine diagnostic testing to confirm ectopic CS was unnecessary and the patient was started on second-line treatment metyrapone immediately when serum cortisol was found to be $3333 \mathrm{nmol} / \mathrm{L}$. This intervention achieved a rapid control of diabetes, normalization of neutrophils and potassium levels and a 
reduction in cortisol levels with significant improvement in symptoms allowing delays in further Cushing's treatment during the COVID pandemic.

\section{Case presentation}

A 65-year-old retired tarmac layer man presented to the emergency department with worsening mobility over the last 6 weeks. He reported unintentional weight loss of 8 $\mathrm{kg}$ over 8 weeks. He was independently mobile and lived with his wife prior to this. He was an ex-smoker (stopped 3 months previously). He had a background medical history of T2DM which had been diagnosed 4 months previously when he was referred to his GP. He also had a history of hypertension which had been difficult to control and a previous history of atrial fibrillation and ischaemic heart disease. His medications were atorvastatin, ramipril, doxazosin, gliclazide, metformin, bisoprolol and sublingual glyceryl trinitrate.

On examination, his temperature was $36.8^{\circ} \mathrm{C}$, BP $178 / 115 \mathrm{mmHg}$, pulse rate $122 \mathrm{bpm}$, weight $89.6 \mathrm{Kg}$ and BMI $28.2 \mathrm{~kg} / \mathrm{m}^{2}$. The patient had Cushingoid features, including a round face, interscapular adiposity, wasted upper and lower limbs proximally, thin skin, purple stria and central obesity. He had clinical evidence of bilateral proximal myopathy and multiple bruises were noted on his forearm and legs. Lower limb examination showed wasting of the quadriceps muscles with no fasciculation and knee flexion of $3 / 5$ bilaterally. Sensory examination was normal. Rectal examination was normal as well.

\section{Investigation}

Initial investigations showed his WCC was slightly elevated at $11.8 \times 10^{9}$ and neutrophils $10.4 \times 10^{9}$ and potassium $3.2 \mathrm{mmol} / \mathrm{L}$. Two months previously during admission, potassium levels varied between 2.2 to 3.4 $\mathrm{mmol} / \mathrm{L}$ and neutrophils were $12.4 \times 10^{9}$. COVID swabs were repetitively negative during the admission.
In view of the lower limb symptoms and signs, an urgent MRI of spine was requested on admission and this showed involvement of the sacrum by extensive tumour with the involvement of the exiting right and, to a lesser extent left, S2 nerve roots (Fig. 1).

Based on all these findings, CS was suspected. Baseline cortisol was $3333 \mathrm{nmol} / \mathrm{L}$ and ACTH level was $96 \mathrm{ng} / \mathrm{L}$. Routine diagnostic testing, overnight dexamethasone suppression test and 24-h urine cortisol were unnecessary (1). CXR showed a suspicious lesion in the right lung and CT of thorax, abdomen and pelvis showed two irregular nodules in the lung's right lower lobe which is highly suspicious for primary lung malignancy, with a pleural-based mass invading the chest wall consistent with metastasis. There was a $67 \mathrm{~mm}$ left adrenal gland metastases. Right adrenal gland was hyperplastic. An ultrasound-guided biopsy from the pleural mass showed an ACTH secreting metastatic small cell lung cancer confirmed by histology.

\section{Treatment}

Oral metyrapone $1000 \mathrm{mg}$ three times per day was started, and within $24 \mathrm{~h}$ potassium levels went back to the normal range and blood glucose levels dropped significantly to an extent that gliclazide had to be stopped to avoid hypoglycaemia (Fig. 2). Blood pressure also improved to readings within normal (Table 1). Neutrophils normalized.

Cortisol levels improved significantly varying between 200 and $500 \mathrm{nmol} / \mathrm{L}$ initially on $1000 \mathrm{mg} 8 \mathrm{~h}$, with metyrapone then titrated up to $1000 \mathrm{mg} 6 \mathrm{~h}$ followed by a block replacement regime adding on hydrocortisone $10 \mathrm{mg}$ three times per day. He was also put-on prophylaxis for Pneumocystis jirovecii with oral co-trimoxazole 960 $\mathrm{mg} /$ day in view of immunosuppression risk and a low-molecular-weight heparin dalteparin 5000 units subcutaneously daily in view of thromboembolism risk.

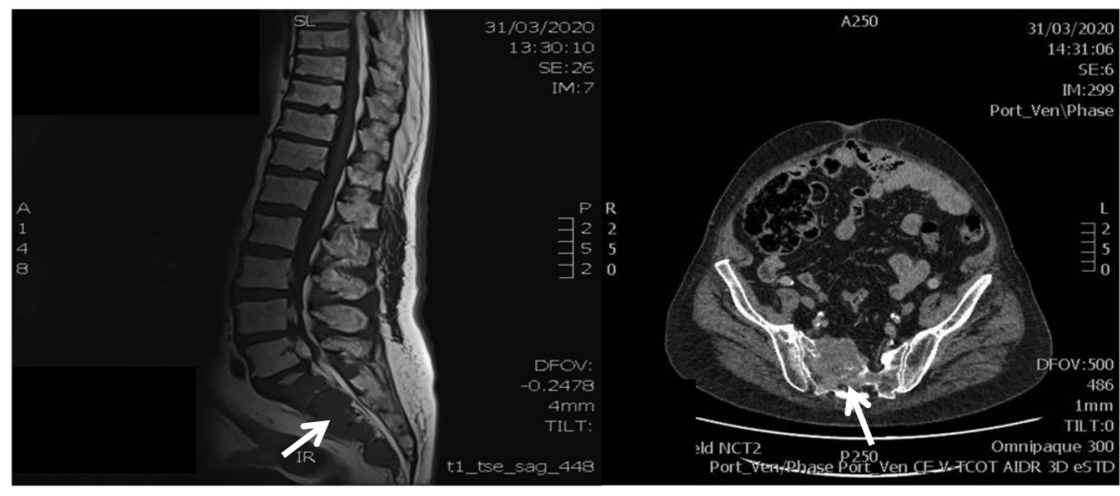

Figure 1

Small cell lung metastasis to sacrum by extensive tumor, principally involving the vertebral bodies, but also extending into the pedicle and lamina of S2 (right), with expansion along the anterior margin of the sacrum into the presacral space, particularly on the right (left). 


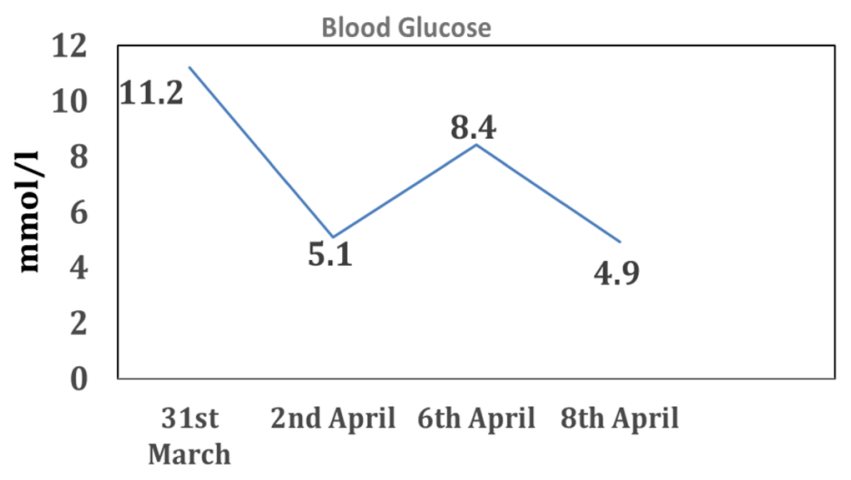

Figure 2

Blood glucose response to metyrapone. Metyrapone was started on the 1 st of April, and gliclazide had to be stopped the next day to avoid hypoglycaemia.

\section{Outcome and follow-up}

For the first few weeks, the patient felt better and was transferred to oncological care. Potassium and neutrophil counts remained within the normal range whilst glucose and BP were controlled. Unfortunately, due to the advanced metastatic small cell lung cancer, the patient passed away weeks later.

\section{Discussion}

The COVID-19 pandemic has hugely influenced medical practice. Following formal guidelines to attain perfection is not generally possible so authorities have designed more appropriate protocols to be used during the pandemic. These recommendations aim to maintain social isolation and lockdown restrictions by reducing out-patient visits, imaging, and biochemical testing. A typical condition influenced by these changes has been Cushing's syndrome (CS). Patients with suspected or confirmed CS usually undergo complex investigations and management, therefore, active management has been recommended for only patients with clear discriminating features of Cushing's and those with moderate to severe features. Importantly, those with diabetes and high blood pressure

Table 1 BP readings during admission and the response to metyrapone.

\begin{tabular}{|c|c|}
\hline Date & BP readings \\
\hline 31st of March & $178 / 115 \mathrm{~mm} \mathrm{Hg}$ \\
\hline 1st of April & 135/100 mm Hg \\
\hline Metyrapone started & \\
\hline 2nd of April & 156/98 mm Hg \\
\hline 6th of April & 112/77 mm Hg \\
\hline 8th of April & 116/80 mm Hg \\
\hline
\end{tabular}

should be treated actively (1). In addition, in patients with severe clinical signs of CS, who are not stressed for example, septic, as opposed to carry out dexamethasone suppression tests and 24-h urine cortisol, a serum cortisol which is very high $>1000 \mathrm{nmol} / \mathrm{L}$ is sufficient to make a diagnosis (1).

Treatment of CS is aimed to reduce its complications. As per Endocrine Society guidelines, the first line treatment of CS is surgical but, in some circumstances, if a patient is not fit for surgery or as part of preoperative preparation, medical treatment can be used first line. In view of COVID restrictions, surgery as a first-line therapeutic strategy as expected is not viable (2). Medical therapy, usually a second line option, could temporarily be used to provide rapid reduction in cortisol levels and allow a safe delay in surgery and possibly achieve good control of complications.

This case report highlights the importance of having a low threshold for suspicion and investigation for CS in a patient with chronic neutrophilia and hypokalaemia, recently diagnosed with type 2 diabetes especially in someone with catabolic features of the disease irrespective of losing weight. It also supports the use of alternative methods of approaching the diagnosis and treatment of CS during a pandemic.

A common cause for ectopic CS is small cell lung cancer (SCLC). CS is a clinical disorder resulting from high levels of circulating glucocorticoids, with concomitant loss of the negative feedback mechanism of cortisol on the hypothalamic-pituitary-adrenal axis (HPA) (3). Ectopic ACTH syndrome is responsible for one-fifth of the ACTH dependent CS, most arise from SCLC or bronchial carcinoid, though any endocrine tumor can cause ectopic ACTH CS (4).

CS can present clinically with a wide range of signs and symptoms including weight gain, lethargy, hirsutism, acne, purple abdominal stria, depression, and muscle weakness. Though the presentation can be nonspecific, certain clinical features are highly suggestive of CS such as proximal myopathy, easy bruising, facial plethora, and abdominal striae. In patients who are not phenotypically cushingoid (e.g. slim rather than overweight), the suspicion should be high for a malignancy-secreting ACTH, especially in the presence of pronounced myopathy, neutrophilia, and profound hypokalaemia (5).

Our patient presented with weight loss. Weight loss is atypical in CS with obesity present in around $85 \%$ of patients in Cushing's disease (6), and the rest is usually overweight. Obesity is lower in ectopic ACTH syndrome occurring in $66 \%$ but only $10 \%$ lose weight so high 
clinical suspicion is necessary (7). Importantly, one should recognize the effect of advanced malignancy as a cause of weight loss in some patients with ectopic ACTH syndrome and this confounds the classical clinical features of CS.

Two-thirds of patients with CS have diabetes and $50 \%$ have impaired glucose metabolism (8). In ectopic CS, diabetes is present in 59\% (7). Hypertension is also common in CS. Hypertension is present in 68\% of patients with Cushing's disease (6), and 59\% of patients with ectopic disease (7).

Both surgery and excess glucocorticoids are known to cause immunosuppression and increase the risk of thromboembolic events (1). So making a quick diagnosis and controlling glucocorticoid excess rapidly reduce the risks of such complications and prevents long periods of admission; this is ideal during the COVID19 pandemic in a patient with CS.

Our patient presented with new-onset diabetes and this together with neutrophilia and hypokalaemia should alert physicians to the possible presence of hypercortisolism. Chronic neutrophilia with hypokalaemia is highly suggestive of hypercortisolism and this pair of findings with new-onset diabetes should alert the general physicians of the possibility of Cushing's syndrome. When the patient presented to us with a cortisol level $>1000 \mathrm{nmol} / \mathrm{L}$ and a high ACTH level with clear features of CS, we were able to make a rapid diagnosis supported by new guidelines "Endocrinology in the time of Covid-19: management of Cushing's syndrome" (1) and avoid extra dexamethasone suppression testing and 24-h urine cortisol tests which would have delayed diagnosis confirmation and treatment.

Treatment options for CS as per Endocrine Society Guidelines recommendations include surgery as a firstline treatment for pituitary lesions responsible for Cushing's disease, for ectopic ACTH-secreting tumors or for unilateral or bilateral adrenal tumors. During COVID, in view of social isolation restrictions to try and reduce exposure time to medical services especially in conditions where patients are immunosuppressed, surgery might not always be the best option. Medical treatments are possible though to try and achieve rapid control of the disease and its complications.

Metyrapone is one of the first-line options for medical treatment. Metyrapone works by reducing cortisol synthesis through inhibition of $11 \beta$ hydroxylase, it also blocks 18 hydroxylase. Metyrapone is given orally and has a short half-life, so it should be given 3-4 times a day, and it is well tolerated (9). In one study looking at the short- and long-term response to metyrapone in patients with CS, 75\% showed biochemical normalization in less than 4 months and 70-83\% had long-term response (10). Metyrapone, in our case, helped to control hypokalaemia, hyperglycaemia and hypertension rapidly during the COVID-19 pandemic. This highlights the effectiveness of this drug in treating CS at least temporarily.

In this case report, we describe the management of a patient who was admitted with ectopic ACTH syndrome during the COVID pandemic with new-onset type 2 diabetes, neutrophilia and unexplained hypokalaemia. These three findings when combined should alert physicians to the potential presence of Cushing's syndrome. On admission, a quick diagnosis of CS was made based on clinical and biochemical features and patient was treated urgently using high dose metyrapone thus allowing delays in surgery and rapidly improving the patient's clinical condition. This resulted in the treatment of hyperglycaemia, hypokalaemia and hypertension reducing cardiovascular risk and likely risk for infection. Following guidelines to treat patients with CS have shown to be effective and offer endocrinologists an option to manage these patients adequately in difficult times.

\section{Declaration of interest}

The authors declare that there is no conflict of interest that could be perceived as prejudicing the impartiality of the research reported.

\section{Funding}

This research did not receive any specific grant from any funding agency in the public, commercial or not-for-profit sector.

\section{Patient consent}

Written informed consent has been obtained from this patient's wife for publication of the submitted article and accompanying images.

\section{Author contribution statement}

M D and Z F managed patient's clinical care, obtained consent and designed the manuscript.

\section{References}

1 Newell-Price J, Nieman LK, Reincke M \& Tabarin A. Endocrinology in the time of COVID-19: management of Cushing's syndrome. European Journal of Endocrinology 2020183 G1-G7. (https://doi. org/10.1530/EJE-20-0352)

2 Nieman LK, Biller BM, Findling JW, Murad MH, Newell-Price J, Savage MO, Tabarin A \& Endocrine Society. Treatment of Cushing's syndrome: an Endocrine Society clinical practice guideline. Journal of Clinical Endocrinology and Metabolism 2015100 2807-2831. (https:// doi.org/10.1210/jc.2015-1818) 
3 Newell-Price J, Bertagna X, Grossman AB \& Nieman LK. Cushing's syndrome. Lancet 2006367 1605-1617. (https://doi.org/10.1016/ S0140-6736(06)68699-6)

4 Ilias I, Torpy DJ, Pacak K, Mullen N, Wesley RA \& Nieman LK. Cushing's syndrome due to ectopic corticotropin secretion: twenty years' experience at the National Institutes of Health. Journal of Clinical Endocrinology and Metabolism 200590 4955-4962. (https:// doi.org/10.1210/jc.2004-2527)

5 Newell-Price J, Trainer P, Besser M \& Grossman A. The diagnosis and differential diagnosis of Cushing's syndrome and pseudo-Cushing's states. Endocrine Reviews 199819 647-672. (https://doi.org/10.1210/ edrv.19.5.0346)

6 Pecori Giraldi F, Moro M, Cavagnini F \& Study Group on the Hypothalamo-Pituitary-Adrenal Axis of the Italian Society of Endocrinology. Gender-related differences in the presentation and course of Cushing's disease. Journal of Clinical Endocrinology and Metabolism 200388 1554-1558. (https://doi.org/10.1210/jc.2002021518)
7 Kamp K, Alwani RA, Korpershoek E, Franssen GJ, de Herder WW \& Feelders RA. Prevalence and clinical features of the ectopic ACTH syndrome in patients with gastroenteropancreatic and thoracic neuroendocrine tumors. European Journal of Endocrinology $2016 \mathbf{1 7 4}$ 271-280. (https://doi.org/10.1530/EJE-15-0968)

8 Feelders RA, Pulgar SJ, Kempel A \& Pereira AM. The burden of Cushing's disease: clinical and health-related quality of life aspects. European Journal of Endocrinology 2012167 311-326. (https://doi. org/10.1530/EJE-11-1095)

9 Daniel E \& Newell-Price JD. Therapy of endocrine disease: steroidogenesis enzyme inhibitors in Cushing's syndrome. European Journal of Endocrinology 2015172 R263-R280. (https://doi. org/10.1530/EJE-14-1014)

10 Verhelst JA, Trainer PJ, Howlett TA, Perry L, Rees LH, Grossman AB, Wass JA \& Besser GM. Short and long-term responses to metyrapone in the medical management of 91 patients with Cushing's syndrome. Clinical Endocrinology 199135 169-178. (https://doi. org/10.1111/j.1365-2265.1991.tb03517.x)

Received in final form 20 April 2021

Accepted 27 April 2021 